Original Paper $\quad$ http://ajol.info/index.php/ijbcs $\quad$ http://indexmedicus.afro.who.int

\title{
Variabilité morphologique de Balanites aegyptiaca (L.) Del. dans la région du Ouaddaï au Tchad
}

\author{
B. ABDOULAYE ${ }^{1}$, A.B. BÉCHIR ${ }^{2 *}$ et P.M. MAPONGMETSEM ${ }^{1}$ \\ ${ }^{1}$ Université de Ngaoundéré, Faculté des Sciences, B.P. 454 Ngaoundéré, Cameroun. \\ ${ }^{2}$ Université des Sciences et de Technologie d'Ati, BP 9957, Ati, Tchad. \\ *Auteur correspondant ; E-mail : abrabechir@yahoo.fr; Tel. : (+235) 662542 61/(+235) 91003574.
}

\section{RESUME}

Dans le Sahel tchadien, le Balanites aegyptiaca est un arbre de grandes importances socioéconomiques pour les populations. L'objectif de l'étude a été de développer les stratégies de conservation et d'utilisation durable des ressources génétiques de l'espèce. Une enquête socio-économique et un inventaire botanique ont été réalisés auprès de 4 groupes ethniques répartis dans 3 villages. Les analyses botaniques ont porté sur 16 descripteurs morphologiques quantitatifs. Les populations locales ont une bonne connaissance de l'espèce et disposent d'un système de classification empirique utilisant des critères communs ou variables selon le groupe ethnique. Les critères communs aux 4 groupes ont été la taille de l'arbre, les dimensions et la forme du fruit, le goût de la pulpe et la taille des feuilles. Le fruit a été de loin le caractère le plus discriminant. Les descripteurs morphologiques discriminants ont été la longueur du grand axe du fruit $(\mathrm{p}<0,001)$, la largeur des feuilles $(\mathrm{p}<0,05)$ et la hauteur de l'arbre $(\mathrm{p}<0,05)$. Trois morphotypes ont été identifié chez l'espèce dont le plus intéressant regroupe des arbres de grande taille, aux feuilles larges et produisant de longs fruits. Ces informations ouvrent de meilleures perspectives pour la sélection variétale et la domestication de cette espèce dans la zone.

(C) 2016 International Formulae Group. All rights reserved.

Mots clés : Fruitiers locaux, Balanites aegyptiaca, savoirs endogènes, morphotypes, Ouaddaï, Tchad.

\section{Morphological variability of Balanites aegyptiaca (L.) Del. in the region of Ouaddaï in Chad}

\begin{abstract}
Balanites aegyptiaca is a tree of high socioeconomic importance for the population who lives in the Sahelian region of Chad. The aim of this study was to develop any strategies of preservation and sustainable use of the genetic resources of the species. Thus, a socioeconomic survey was carried out within four ethnic groups in three villages and botanical inventory was set up for the purpose. The botanical analyses dealt with 16 quantitative morphological descriptors. The targeted communities have a good knowledge of the species and make use of and empirical classification system based on common or variable criteria according to the ethnical groups under study. The common criteria to the 4 groups were the height of the tree, the size and shape of the fruit, the pulp taste and the length of the leaf. The fruit was by far the most discriminating aspect. The discriminating morphological descriptors were the length of the main axis of the fruit $(\mathrm{p}<0.001)$, the width of
\end{abstract}


the leaf $(\mathrm{p}<0.05)$ and the height of the tree $(\mathrm{p}<0.05)$. Three morphotypes were identified in the breed, the most interesting of which concerns high, wide-leafed and long fruit producing trees. This information paves the way for better prospects as far as the selection of varieties and the domestication of the species in the area are concerned.

(C) 2016 International Formulae Group. All rights reserved.

Keywords: Local fruit-trees, Balanites aegyptiaca, endogenous knowledge, morphotypes, Ouaddaï, Chad.

\section{INTRODUCTION}

En plus du déficit pluviométrique observé ces dernières décennies dans la région du Ouaddaï en zone sahélienne, est venue s'ajouter la pression démographique due à l'installation des camps des réfugiés et déplacés des conflits du Darfour. Cette situation s'est traduite par la dégradation excessive des ressources naturelles. De nombreuses espèces ligneuses se trouvent ainsi aujourd'hui menacées. C'est pourquoi, il est urgent d'agir pour freiner sinon stabiliser cette dégradation pour les générations futures. Balanites aegyptiaca (L.) Del. est un arbre cultivé en Egypte depuis la haute antiquité. C'est une espèce des zones sahéliennes, soudano-sahéliennes d'Afrique, de la Péninsule Arabique, de l'Inde jusqu'au Pakistan (Eyog et al., 2000; Arbonnier, 2004). L'arbre est utilisé dans l'alimentation humaine et comme fourrage (Elseed et al., 2002). L'un des arbres fruitiers prioritaires pour les communautés rurales dans le Sahel ouest-africain, le B. aegyptiaca (L.) Del. joue un rôle multifonctionnel dans la vie quotidienne des populations (Sagna et al., 2014). Sa valeur principale réside dans sa parfaite adaptation aux écosystèmes arides (Chevalier et al., 2004) et une bonne production de fruits qui sont consommés surtout en période de pénurie alimentaire et commercialisés sur les marchés des villes et villages (Makalao et al., 2015). B. aegyptiaca (L.) Del. est l'une des espèces alimentaires locales les plus prisées mais malheureusement vulnérable (Mapongmetsem et al., 2012). Les valeurs nutritionnelles de ces fruits sont également rapportées par plusieurs auteurs (Elfeel, 2010; Sagna et al., 2014). Surexploitée, menacée par les feux, le surpâturage et la sécheresse, cette essence est en nette régression (FAO, 2001). C'est pourquoi, l'étude de sa variabilité morphologique constitue une étape indispensable pour sa domestication. Cela exige donc la priorisation des préférences des populations locales et la prise en compte de leur expérience en matière de conservation et de valorisation des plantes. La domestication de l'espèce nécessite également l'identification de bonnes productrices de graines, la connaissance des caractéristiques morphologiques et le développement des techniques de propagation (Mapongmetsem et al., 2011). Cependant, la meilleure connaissance de cet arbre, son utilisation et sa gestion durable demandent préalablement une caractérisation morphologique de ses matériels génétiques. En milieu rural africain, les populations disposent de connaissances sur les plantes et utilisent différents critères pour les caractériser (Assogbadjo et al., 2008). Au Tchad, très peu de données sur les connaissances écologiques locales de $B$. aegyptiaca (L.) Del. existent. L'objectif de ce travail est donc de contribuer au développement des stratégies de conservation et d'utilisation durable de ce patrimoine naturel en évaluant la variabilité morphologique de B. aegyptiaca (L.) Del., objet de la présente étude.

\section{MATERIEL ET METHODES}

Présentation de la zone d'étude

L'étude a été effectuée dans la région du Ouaddaï dans le Tchad oriental. Les investigations ont concerné trois (3) villages de la région: Arkou $\left(13^{\circ} 52^{\prime} \mathrm{N}\right.$ et $\left.20^{\circ} 49^{\prime} \mathrm{E}\right)$, Kokorguin $\left(13^{\circ} 29^{\prime}\right.$ de latitude Nord et $20^{\circ}$ $39^{\prime}$ de longitude Est) et Mandafana (13० $54^{\prime}$ de latitude Nord et $20^{\circ} 42^{\prime}$ de longitude Est) (Figure 1). Située entre le $12^{\text {ème }}$ et le $16^{\text {ème }}$ 
parallèle Nord avec une superficie de 29980 $\mathrm{km}^{2}$, la zone d'étude compte 731679 habitants (INSEED, 2009). Comprise entre les isohyètes 200 à $600 \mathrm{~mm}$, cette zone est sous l'influence d'un climat de type sahélien avec une longue saison sèche (novembre à mai) et une courte saison des pluies (de juin à octobre). La pluviométrie moyenne annuelle se situe entre $200 \mathrm{~mm}$ au Nord et $950 \mathrm{~mm}$ au Sud.

La végétation suit le gradient pluviométrique Nord/Sud et se présente sous la forme de steppes herbeuses, arbustives basses à épineux au Nord, à de steppes arborées et des forêts claires ou peu denses à moyennement denses au Sud. Les « ouaddis» $\mathrm{du}$ Nord-Ouest sont caractérisés par une végétation arbustive souvent claire composée d'espèces comme Acacia tortilis, Acacia mellifera, Acacia laeta, Balanites aegyptiaca, Boscia senegalensis, Dichrostachys cinerea, Ziziphus mauritiana, Sclerocarya birrea, et Salvadora persica. Au Sud sur sols argileux, la végétation devient plus boisée. Tamarindus indica, Anogeissus leiocarpus et Combretum glutinosum forment des forêts galeries assez denses. Les sols sableux à sablo-argileux de fonds des vallées sont en revanche occupés par Acacia seyal et Acacia nilotica. La strate herbacée sous forme de tapis plus ou moins continu est dominée par Schoenefeldia gracilis, Zornia glochidiata, Cassia obtusifolia, Dactyloctenium aegyptiaca.

La température de la région varie selon la saison. Pendant la saison sèche froide (décembre/février), les températures journalières se situent en moyenne entre 16 et $35{ }^{\circ} \mathrm{C}$; en saison sèche chaude (mars/avril), les températures oscillent entre 25 et $41{ }^{\circ} \mathrm{C}$; en saison des pluies (août), elles diminuent et varient entre 21 et $31{ }^{\circ} \mathrm{C}$. Le relief très accidenté est marqué par des affleurements rocheux ainsi que la présence de pierres, de blocs et de rochers. La Région est parcourue par des cours d'eau qui sont en majorité de type non permanent et endoréique, dont la durée des écoulements dépend de la variabilité de la pluviométrie et de leur position dans la Région.
Les données ont été collectées dans 3 villages (Arkou, Kokorguin et Mandafana) de septembre 2012 à février 2013. La Région du Ouaddaï représente le domaine de répartition naturelle de $B$. aegyptiaca avec plusieurs modes de distribution des associations végétales et d'utilisation de terres.

\section{Enquêtes socio-économiques}

Des enquêtes ont été réalisées auprès d'hommes et des femmes des villages concernés par des interviews semi-structurées. Celles-ci reposaient sur un questionnaire testé au préalable et contenant des questions fermées et ouvertes. Les principaux points du questionnaire ont porté sur les savoirs endogènes, les critères utilisés dans la classification traditionnelle, l'état des peuplements et les menaces qui pèsent sur cette espèce. Au total, 172 personnes volontaires (94 hommes et 78 femmes) âgés de 35 à 75 ans ont été interviewées. Ces personnes appartenaient aux groupes ethniques (Maba, Massalit, Mimi et Tama) des villages ciblés (Tableau 1).

Les entretiens ont été réalisés en arabe local ou traduit en langue locale pour faciliter la compréhension. Les interviews individuelles ont été complétées par des entretiens de groupe (5 à 15 personnes). La classification paysanne de cette espèce a été réservée aux personnes des deux sexes âgées de plus de 50 ans, à cause de leur expérience et leur aptitude à différencier des pieds de $B$. aegyptiaca.

\section{Evaluation des descripteurs morphologiques discriminants}

Afin de mieux identifier les individus ciblés, les travaux d'évaluation ont été précédés par des missions d'identification sur le terrain. L'évaluation a concerné 30 pieds en cours de fructification, en raison de 10 individus distants d'au moins $100 \mathrm{~m}$ par site. Plusieurs caractères morphologiques jugés plus discriminants ont été ainsi retenus pour être évalués. 


\section{Descripteurs quantitatifs du tronc}

Le caractère morphologique discriminant retenu au niveau du tronc est le diamètre à hauteur de poitrine $(\mathrm{DBH})$ pris à $1,30 \mathrm{~m} \mathrm{du}$ sol, exprimé en centimètre et mesuré à l'aide d'un ruban métrique de couturier $( \pm 1 \mathrm{~mm})$. Les mesures du tronc ont été effectuées en août 2012.

\section{Descripteur quantitatif du houppier}

Le descripteur quantitatif du houppier est la hauteur de la première branche par rapport au sol. Ce paramètre est indispensable pour la domestication car il représente plus de $70 \%$ du volume des arbres des savanes (Kouyaté, 1995). La mesure de la hauteur de la première grosse branche permet d'identifier des individus aux branches basses facilitant la cueillette des fruits. La hauteur des arbres et celle de la première branche par rapport au sol ont été mesurées à l'aide d'un clinomètre.

\section{Descripteur quantitatif des feuilles}

Les mesures descriptives ont porté sur 10 feuilles fraîches totalement développées et non parasitées par individus soit au total 300 feuilles sur l'ensemble de 30 individus. Les mesures ont concerné la longueur et la largeur des feuilles mesurées à l'aide d'une règle graduée et exprimées en $\mathrm{cm}$. La longueur et la largeur des folioles exprimées en mm ont été mesurées à l'aide d'une règle graduée de 30 $\mathrm{cm}( \pm 1 \mathrm{~mm})$. Le nombre de folioles par feuille a été déterminé par comptage simple.

\section{Descripteur quantitatif du fruit}

Les mensurations ont concerné 10 fruits mûrs non parasités par individu (Figure 2) récoltés en septembre 2012, soit au total 300 fruits. Les récoltes ont été effectuées en secouant les branches ou en grimpant directement sur l'arbre. Les dimensions du grand et du petit axe du fruit ont été déterminées à l'aide d'une règle graduée. La longueur du grand axe du fruit a été mesurée à partir du point d'attache du fruit au pédoncule jusqu'à l'extrémité. Les poids du fruit, de la pulpe et de la graine ont été ensuite mesurés grâce à une balance AND (type HT 300, $\max =300 \mathrm{~g} ; \min =0,5$ $\pm 1 \mathrm{~g})$.

\section{Descripteur quantitatif de l'épine}

Les mesures portées sur la longueur exprimée en centimètre $(\mathrm{cm})$ ont été effectuées sur 10 épines totalement développées par individu soit 300 épines pour l'ensemble des trois sites. Les mensurations ont été effectuées en septembre 2012 et les épines ont été directement mesurées sur le rameau ou dégagées de celui-ci donc isolées.

\section{Descripteur quantitatif de la graine}

Les descripteurs retenus au niveau de la graine ont été le grand et le petit axe déterminés à l'aide d'un pied à coulisse, et le poids mesuré grâce à une balance AND (type HT 300, $\max =300 \mathrm{~g} ; \min =0,5 \pm 1 \mathrm{~g}$ ). Les mesures ont été réalisées en mai 2012 et octobre 2012.

\section{Analyse des données}

Les logiciels STRATIGRAPHICS PLUS et XLSTAT ont été utilisés pour les analyses statistiques. L'analyse de variance (ANOVA) a été utilisée. Les descripteurs significatifs ont été ensuite soumis à l'Analyse en Composante Principale (ACP) et à la classification ascendante hiérarchisée.

\section{RESULTATS}

\section{Classification paysanne des individus}

Les résultats ont montré que $95 \%$ des populations enquêtées disposent d'amples connaissances sur le B. aegyptiaca qu'elles intègrent dans leur vie socio-économique et culturelle. Pour distinguer les morphotypes, elles utilisent des critères botaniques empiriques de différenciation variables selon le groupe ethnique. De façon générale, les Maba connaissent plus de critères de différenciation que les autres groupes ethniques. En effet, pour différencier les individus de B. aegyptiaca, les Maba utilisent 13 critères de différenciation contre $12 \mathrm{chez}$ les Massalit et 10 chez les Mimi et les Tama. Les critères de différenciation communs aux quatre groupes ethniques ont été : la taille de l'arbre, la morphologie, les dimensions des feuilles et des fruits, et le goût de la pulpe. 


\section{Critère taille de l'arbre}

Concernant le critère taille de l'arbre, les Maba (79\%), les Massalit (76\%), les Mimi (71\%) et les Tama (84\%) ont utilisé la hauteur de l'arbre pour différencier les individus de $B$. aegyptiaca. Ils distinguent ainsi des individus adultes de grande et de petite taille.

\section{Critères forme et dimension des feuilles}

Des individus de $B$. aegyptiaca ont été également identifiés par la forme et la taille de leurs feuilles. C'est ainsi que les Maba (71\%) et les Massalit (66\%) reconnaissent des individus à larges feuilles arrondies, des individus à feuilles allongées moyennes et ceux à petites feuilles allongées. Les Mimi $(68 \%)$ et les Tama (73\%) identifient en revanche des individus à larges feuilles arrondies et ceux à petites feuilles allongées (Figure 3).

\section{Critères taille, forme et goût du fruit}

Le fruit a été de loin le critère le plus discriminant pour $81 \%$ des personnes interrogées. Les principaux descripteurs utilisés ont été le goût de la pulpe (amer vs sucré : $44 \%$ ), la taille (gros vs petit : $39 \%$ ) et la forme (allongée $v s$ arrondie : $52 \%$ ) du fruit. En outre, une corrélation a été également faite entre la taille, la forme et le goût du fruit. En effet, $38,4 \%$ des personnes enquêtées affirment que les gros fruits sont généralement plus amers que les petits qui sont le plus souvent doux. Sur cette base, les Massalit (78\%) différencient 4 types morphologiques de $B$. aegyptiaca. Il s'agit d'individus à petits fruits ronds et sucrés, des individus à gros fruits allongés et amers, des individus à gros fruits allongés et sucrés et ceux à petits fruits allongés et amers. Les Mimi (59\%) et les Tama (71\%) ne reconnaissent en revanche que 3 types morphologiques de fruits en se basant sur la grosseur, la forme et le goût de la pulpe. Sur cette base, ils distinguent des individus à gros fruits allongés et sucrés, des individus à petits fruits allongés et amers et ceux à gros fruits allongés et amers (Figure 3). Pour ces deux derniers groupes ethniques, les fruits de $B$. aegyptiaca ont tous une forme allongée. Les Maba (71\%) utilisent les mêmes critères que les Massalit mais ne reconnaissent également que 3 types morphologiques: des individus à petits fruits ronds et sucrés, des individus à gros fruits allongés et sucrés et des individus à petits fruits allongés et amers.

Par ailleurs, 56\% des Maba ont utilisé l'épaisseur de la pulpe comme critère de différenciation. Ce groupe identifie des fruits de $B$. aegyptiaca à pulpe charnue et ceux à pulpe mince.

\section{La longueur et le nombre d'épines comme critères de différenciation}

Sur la base du nombre et de la longueur des épines, les Mimi (51\%), les Massalit (44\%), les Maba (39\%) et les Tama (31\%) distinguent trois (3) morphotypes de $B$. aegyptiaca: des individus aux épines courtes et peu nombreuses, des individus aux épines courtes et très nombreuses et ceux aux épines longues et nombreuses (Figure 3).

\section{La géolocalisation de l'arbre sur le terrain}

La situation de l'arbre sur le terrain est une source d'inspiration pour les populations de la zone. Chez les Maba, $89 \%$ des personnes interrogées affirment que le $B$. aegyptiaca se développement beaucoup plus sur les plaines et que les grands arbres ne sont inféodés qu'au sol sableux. Concernant la situation topographique, $66 \%$ des Maba et $73 \%$ des Massalit ont déclaré que les individus nains colonisent le plus souvent les montagnes (Tableau 2).

\section{Caractérisation botanique de l'espèce}

Les connaissances endogènes des populations de la Région ont motivé le choix des descripteurs morphologiques quantitatifs de l'espèce. Parmi les 16 descripteurs morphologiques discriminants évalués, seuls 3 ont été significatifs : la largeur des feuilles ( $\mathrm{p}$ $<0,05)$, la longueur du grand axe du fruit ( $\mathrm{p}<$ $0,001)$ et la hauteur de l'arbre $(\mathrm{p}<0,05)$.

\section{Caractéristiques morphologique du tronc, la taille et la hauteur de la première grosse branche}

Contrairement aux individus adultes dont la texture du tronc est plus rugueuse, les jeunes individus de B. aegyptiaca de notre 
zone d'étude ont montré un tronc le plus souvent lisse. Le diamètre à hauteur de poitrine $(1,30 \mathrm{~m})$ a varié de $16,63 \mathrm{~cm}$ (à Kokorguin) à 19,09 cm (à Arkou). La moyenne générale a été de 17,60 $\pm 0,07 \mathrm{~cm}$ (Tableau 3). Les individus de B. aegyptiaca ont une hauteur moyenne de 6,35 $\pm 1,27 \mathrm{~m}$. La variation intra-population oscille entre 5,47 et 7,07 m. Cette différence est significative (p $<0,05)$. Les plus grands individus $(7,07 \pm$ $1,35 \mathrm{~m}$ ) ont été mesurés à Mandafana et les plus petits $(5,47 \pm 0,69 \mathrm{~m})$ à Kokorguin. En outre, il existe une corrélation significative et positive entre la longueur du grand axe du fruit et la hauteur générale de l'arbre $(r=$ $0,414 ; \mathrm{p}<0,05)$.

La hauteur moyenne de la première branche vivante chez les populations de $B$. aegyptiaca a été de $2,45 \pm 1,27 \mathrm{~m}$. Cette hauteur a varié entre 1,86 et $2,95 \mathrm{~cm}$.

\section{Dimensions et poids du fruit et de la graine}

Des différences significatives ont été observées entre la dimension des fruits, quelque soit les sites de prélèvement. Les fruits de $B$. aegyptiaca ont mesuré en moyenne 2, $86 \pm 0,30 \mathrm{~cm}$ de long pour l'ensemble des individus des différents sites. La longueur du grand axe des fruits chez l'espèce a varié entre 2,55 et $3,15 \mathrm{~cm}$. La longueur du petit axe du fruit a oscillé entre 1,99 à $2,13 \mathrm{~cm}$ pour une moyenne de 2,05 \pm $0,15 \mathrm{~cm}$. La différence entre les longueurs du grand axe du fruit a été significative ( $\mathrm{p}<$ 0,001 ) alors qu'aucune différence significative n'a été observée entre les fruits des individus des différents sites $(\mathrm{p}>0,05)$. Les plus longs fruits $(3,15 \pm 0,26 \mathrm{~cm})$ ont été observés à Mandafana. En revanche, c'est à Kokorguin que les fruits les plus courts $(2,55 \pm 0,38 \mathrm{~cm})$ ont été notés (Tableau 4).

Le poids des fruits a varié entre 4,90 et $5,04 \mathrm{~g}$ pour un poids moyen de l'ordre de 4,95 $\pm 1,26$ g. Cependant, on n'a pas observé une différence significative entre les poids des différents fruits $(p>0,05)$. La moyenne des poids des pulpes a été de 1,87 $\pm 0,65 \mathrm{~g}$. La variation du poids de la pulpe entre les différents sites a oscillé entre 1,85 et 1,89 g.
Cette différence n'a pas été cependant significative $(\mathrm{p}>0,05)$.

Le poids des graines a varié entre 2,05 et 2,42 $\mathrm{g}$ pour un poids moyen de l'ordre 2,16 $\pm 0,51 \mathrm{~g}$. La différence entre les poids des graines n'a pas été également significative (p> 0,05). Le rapport pondéral pulpe/fruit qui est de $37 \%$ n'a pas significativement varié entre les sites (Tableau 4).

\section{Dimensions des feuilles, des folioles et longueur des épines}

La largeur moyenne des feuilles de l'espèce mesurée dans les 3 sites a été de $27,11 \pm 1,43 \mathrm{~mm}$. Les plus larges feuilles ont été mesurées à Arkou $(28,11 \pm 1,43 \mathrm{~mm})$ alors que les plus étroites l'ont été à Kokorguin $(25,65 \pm 0,86 \mathrm{~mm})$. Les résultats de la caractérisation des folioles ont montré que la longueur moyenne a varié entre 35,54 et 36,02 $\mathrm{mm}$. Cependant, cette variation n'est pas importante car les individus ne sont pas significativement différents $(p>0,05)$. La largeur des folioles a variée en revanche de 25,65 mm (site de Kokorguin) à 28,52 mm (site d'Arkou). Cette variation n'est pas non plus significative $(p>0,05)$. La surface foliaire suit également la même tendance (Tableau 5). De même, la largeur de la feuille est corrélée significativement et positivement à la longueur du fruit $(r=0,39 ; p<0,05)$.

La longueur des épines a variée entre 31,15 et $32,69 \mathrm{~mm}$ pour une longueur moyenne de 31, $84 \pm 4,03 \mathrm{~mm}$. Concernant le critère longueur des épines, la différence n'a pas été également significative $(\mathrm{p}>0,05)$.

\section{Analyse en Composantes Principales}

L'essentiel de l'information du tableau de contingence est contenu dans les trois principaux axes factoriels. Le plan factoriel constitué des axes F1 et F2 explique $85 \%$ de la totalité des variables analysées (Figure 4). Avec respectivement $52 \%$ et $33 \%$, de variance expliquée, la représentation du premier plan factoriel a montré que positivement, la largeur de la feuille et la hauteur de l'arbre interviennent fortement dans la création de l'axe F1. La distribution des individus de $B$. 
aegyptiaca est hétérogène comme en témoignent la répartition des nuages de points qui se regroupent autour de deux variables qui sont, la largeur de la feuille et la hauteur de l'arbre. Par contre, le troisième nuage n'est lié à aucune des variables étudiées. Ces nuages de points sont composés de la manière suivante : le premier regroupe d'une part, les individus 2, 3, 8, 9 et 10 de Mandafana et, d'autre part, les individus 10 et 4 d'Arkou et de Kokorguin respectivement. Le deuxième nuage discrimine les individus 3,5 et 8 d'Arkou et les arbres 1, 4, 5, 6 et 7 de Mandafana. Le troisième nuage regroupe les individus 1, 7 et 9 d'Arkou et 1, 2, 3, 5, 6, 9 et 10 de Kokorguin. Ceux-ci n'ont aucune corrélation avec les variables étudiées (Figure 4).

Une analyse plus approfondie à partir de la classification hiérarchisée a permis de mettre au point un dendrogramme (Figure 5) regroupant 30 pieds de $B$. aegyptiaca. L'indice critique de similarité est situé à 0,91 .
A cette zone de coupe, on note l'existence de 3 morphotypes de B. aegyptiaca dans la zone au sein desquels se retrouvent des sousgroupes plus ou moins importants (Figure 5) :

- le premier morphotype représente $26,66 \%$ de l'effectif global. Les individus sont de petite taille $(4,55 \pm 0,82 \mathrm{~m})$, aux feuilles larges $(62,8 \pm 0,67 \mathrm{~mm})$ et produisent de petits fruits $(2,76 \pm 0,44 \mathrm{~cm})$;

- le deuxième morphotype est constitué d'individus de grande taille $(8,2 \pm$ $1,13 \mathrm{~m})$ et contrairement au précédant morphotype, leurs feuilles sont de petites dimensions $(47,6 \pm 0,65 \mathrm{~mm})$ et produisent de longs fruits $(2,91 \pm 0,3 \mathrm{~cm})$. Ce morphotype est bien représenté dans la zone $(43,33 \%)$;

- le troisième morphotype regroupe les arbres de taille moyenne $(5,80 \pm 0,80 \mathrm{~m})$ qui portent de larges feuilles $(55,50 \pm 1,07 \mathrm{~mm})$ et produisant de longs fruits $(2,86 \pm 0,40 \mathrm{~cm})$. Les individus renfermant ces caractères constituent $30 \%$ de l'effectif.

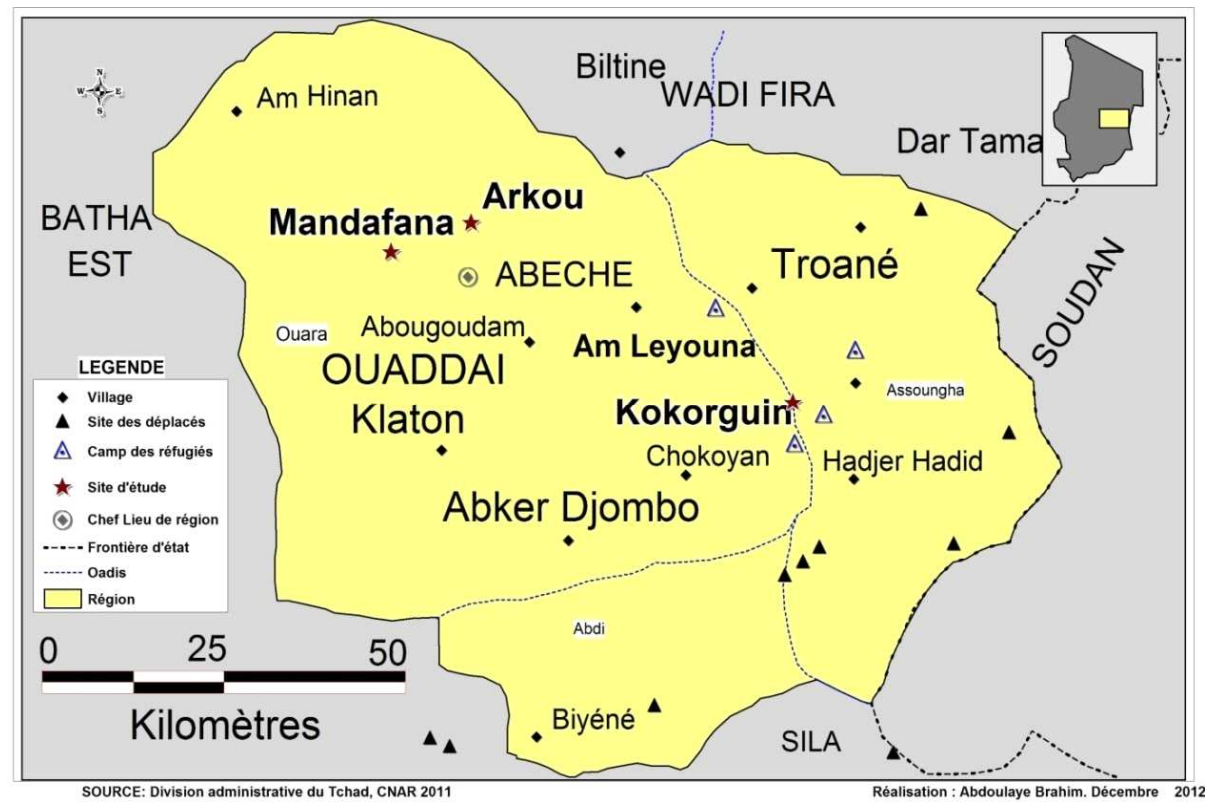

Figue 1 : Localisation des lieux d'étude. 

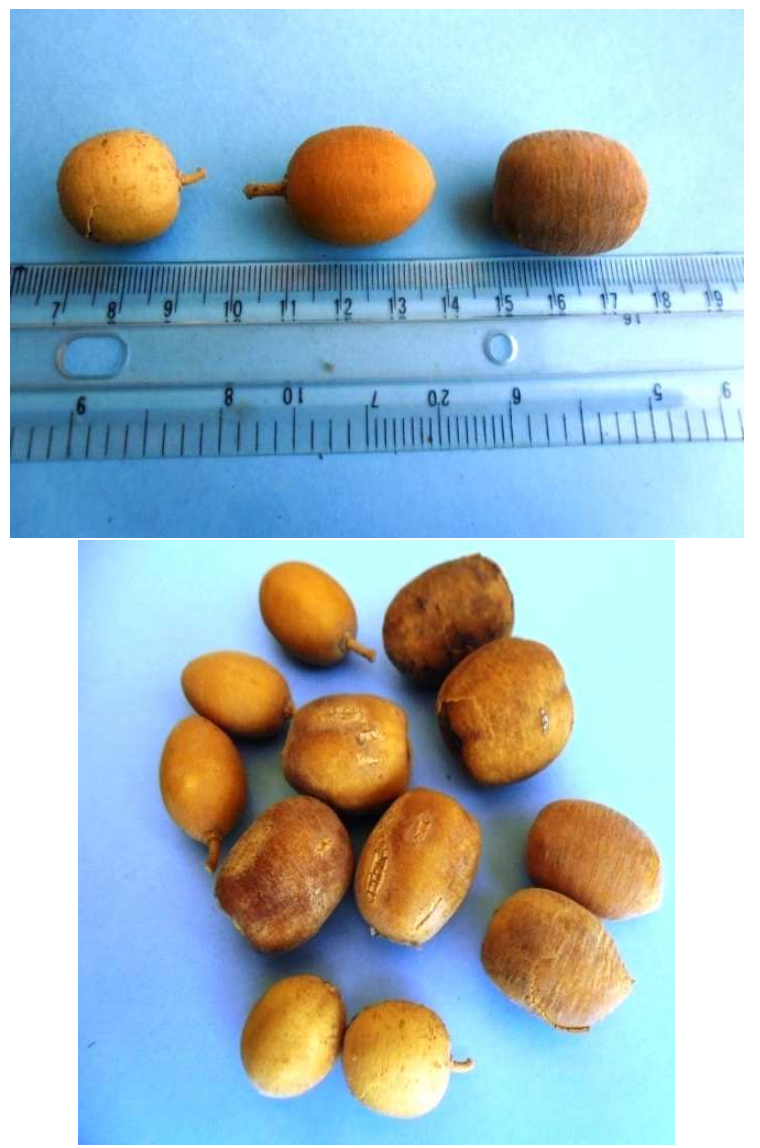

Figure 2 : Différents types de fruits de B. aegyptiaca.

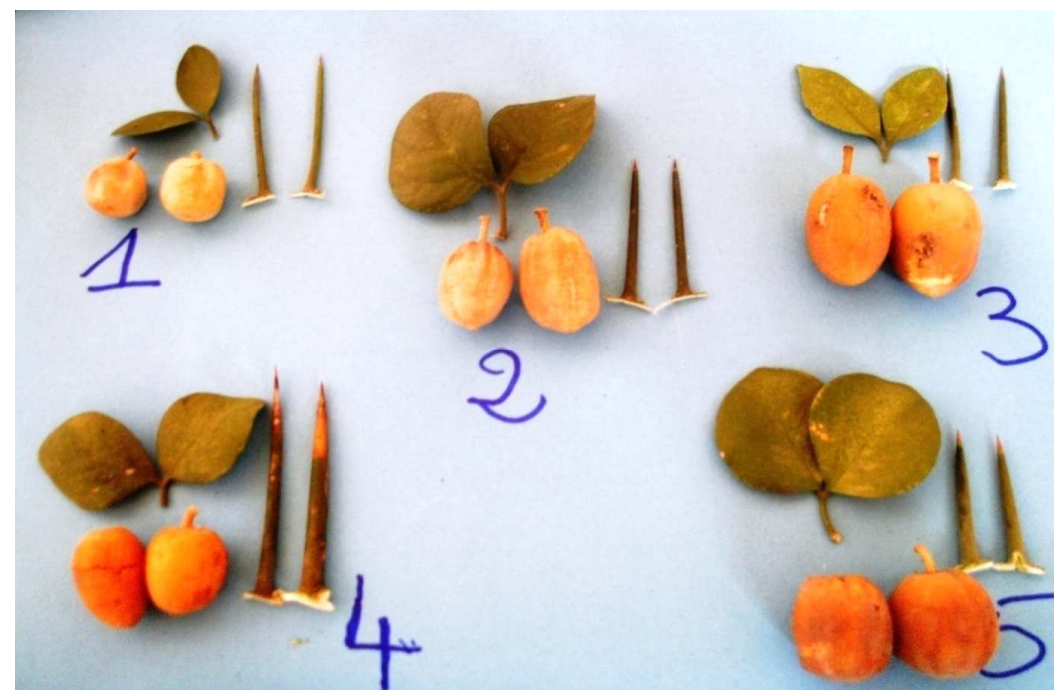

Figure 3 : Classification de B. aegyptiaca suivant les organes, selon les Maba. 


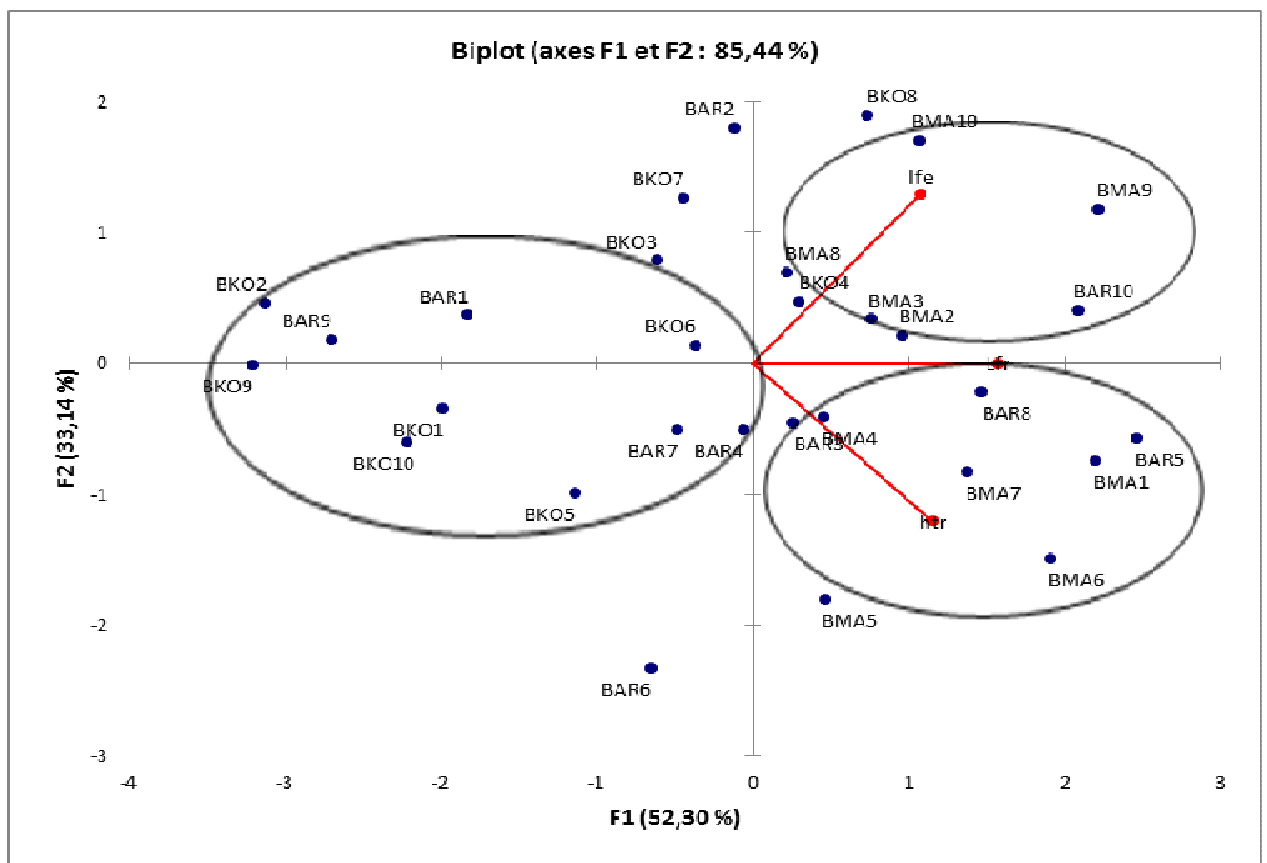

Figure 4 : Distribution des individus dans le plan $F_{1} \times F_{2}$

$\mathrm{BKO}=$ Balanites Kokorguin $; \mathrm{BAR}=$ Balanites Arkou $; \mathrm{BMA}=$ Balanites Mandafana .

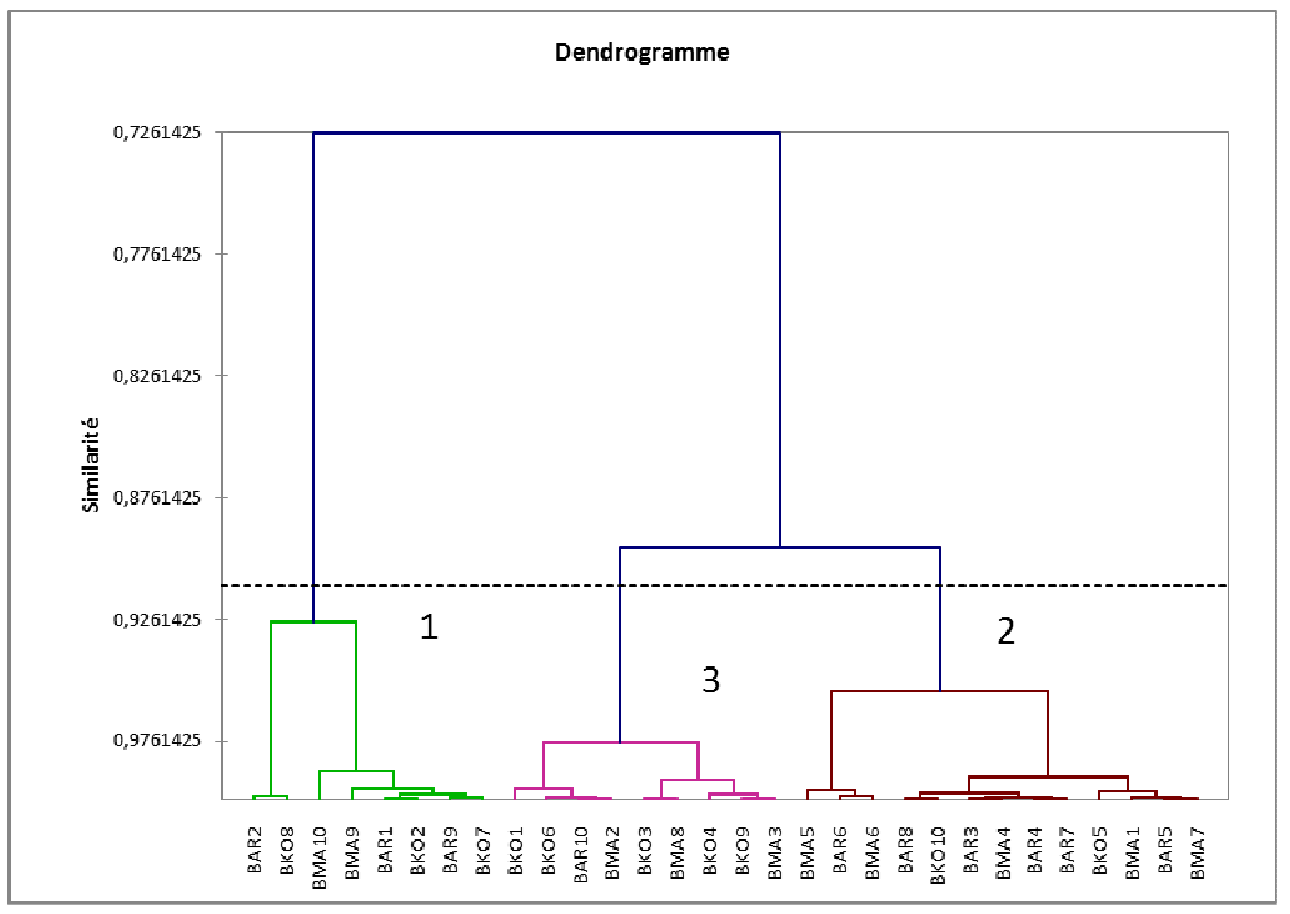

Figure 5 : Dendrogramme de B. aegyptiaca.

$\mathrm{BAR}=$ Balanites Arkou $; \mathrm{BKO}=$ Balanites Kokorguin $; \mathrm{BMA}=$ Balanites Mandafana . 
Tableau 1 : Répartition des personnes enquêtées selon l'ethnie et le sexe.

\begin{tabular}{lccccc}
\hline \multicolumn{7}{c}{ Ethnies } \\
\hline & Maba & Massalit & Mimi & Tama & Total \\
\hline Ménages & 20 & 17 & 17 & 18 & $\mathbf{7 2}$ \\
Hommes & 27 & 22 & 21 & 24 & $\mathbf{9 4}$ \\
Femmes & 21 & 18 & 20 & 19 & $\mathbf{7 8}$ \\
Total & $\mathbf{4 8}$ & $\mathbf{4 0}$ & $\mathbf{4 1}$ & $\mathbf{4 3}$ & $\mathbf{1 7 2}$ \\
\hline
\end{tabular}

Tableau 2 : Classification paysanne de Balanites aegyptiaca suivant les critères.

\begin{tabular}{|c|c|c|c|c|c|}
\hline $\begin{array}{l}\text { Critères ou organes } \\
\text { d'identification }\end{array}$ & Critères & Variantes & \multicolumn{3}{|c|}{ Pourcentage des répondants } \\
\hline \multirow{4}{*}{$\begin{array}{l}\text { Taille de l'arbre } \\
\text { Emplacement de } \\
\text { l'arbre }\end{array}$} & \multirow{4}{*}{$\begin{array}{c}\text { Hauteur } \\
\text { Géolocalisation }\end{array}$} & Grande & Maba $76 \%$ & Massalit & $79 \%$ \\
\hline & & Petite & Mimi $71 \%$ & Tama & $84 \%$ \\
\hline & & Plaine & Maba $89 \%$ & Massalit & $63 \%$ \\
\hline & & Montagne & Mimi 58\% & Tama & $61 \%$ \\
\hline \multirow{6}{*}{ Fruit } & \multirow{3}{*}{ Forme } & Allongée & Maba $71 \%$ & Massalit & $66 \%$ \\
\hline & & Arrondie & Mimi 68\% & Tama & $73 \%$ \\
\hline & & Gros & Maba $71 \%$ & Massalit & $78 \%$ \\
\hline & \multirow[t]{2}{*}{ Grosseur } & Petit & Mimi 59\% & Tama & $71 \%$ \\
\hline & & sucré & Maba $41 \%$ & Massalit & $68 \%$ \\
\hline & Saveur & Amère & Mimi $49 \%$ & Tama & $48 \%$ \\
\hline \multirow{4}{*}{ Feuilles } & \multirow[t]{2}{*}{ Forme } & Allongées & Mimi 49\% & Tama & $48 \%$ \\
\hline & & Arrondies & Maba 66\% & Massalit & $71 \%$ \\
\hline & Dimensions & Larges & Mimi 68\% & Tama & $73 \%$ \\
\hline & & Petites & & • $\quad$ - & \\
\hline \multirow[b]{2}{*}{ Folioles } & \multirow{3}{*}{ Dimensions } & Grandes & Maba $11 \%$ & Massalit & $21 \%$ \\
\hline & & Moyennes & Mimi $18 \%$ & Tama & $43 \%$ \\
\hline \multirow{4}{*}{ Epines } & & Nombreuses & Maba 39\% & Massalit & $44 \%$ \\
\hline & \multirow[t]{2}{*}{ Nombre } & peu & Mimi 51\% & Tama & $31 \%$ \\
\hline & & Longues & Maba $39 \%$ & Massalit & $44 \%$ \\
\hline & Longueur & Courtes & Mimi 51\% & Tama & $31 \%$ \\
\hline
\end{tabular}

Tableau 3 : Taille, hauteur moyenne de la première grosse branche et DBH de B. aegyptiaca selon le site.

\begin{tabular}{|c|c|c|c|c|}
\hline & \multicolumn{4}{|c|}{ Sites } \\
\hline Descripteur morphologique & Arkou & Kokorguin & Mandafana & Moyenne \\
\hline Hauteur de la $1^{\text {ere }}$ branche $(\mathrm{m})$ & $2,56 \pm 1,29$ & $1,86 \pm 1,20$ & $2,95 \pm 1,32$ & $2,45 \pm 0,07$ \\
\hline DBH $(\mathrm{cm})$ & $19,09 \pm 0,06$ & $16,63 \pm 0,08$ & $17,10 \pm 0,08$ & $17,60 \pm 0,07$ \\
\hline Taille de l'arbre (m) & $6,52 \pm 1,78$ & $5,47 \pm 0,69$ & $7,07 \pm 1,35$ & $6,35 \pm 1,27$ \\
\hline
\end{tabular}

$\mathrm{BDH}=$ Diamètre à Hauteur de Poitrine 
Tableau 4 : Caractéristiques des fruits et de la graine de B. aegyptiaca selon les sites.

\begin{tabular}{lcccc}
\hline \multicolumn{4}{c}{ Sites } \\
\hline Descripteur morphologique & Arkou & Kokorguin & Mandafana & Moyenne \\
\hline Lxf $(\mathrm{cm})$ & $2,89 \pm 0,26$ & $2,55 \pm 0,38$ & $3,15 \pm 0,26$ & $2,86 \pm 0,30$ \\
$\operatorname{lxf}(\mathrm{cm})$ & $1,99 \pm 0,15$ & $2,04 \pm 0,20$ & $2,13 \pm 0,12$ & $2,05 \pm 0,15$ \\
Pfr $(\mathrm{g})$ & $5,04 \pm 1,19$ & $4,90 \pm 1,87$ & $4,91 \pm 0,74$ & $4,95 \pm 1,26$ \\
Ppu (g) & $1,88 \pm 0,72$ & $1,89 \pm 0,68$ & $1,85 \pm 0,56$ & $1,87 \pm 0,65$ \\
Pgr $(\mathrm{g})$ & $2,05 \pm 0,60$ & $2,06 \pm 0,62$ & $2,42 \pm 0,32$ & $2,16 \pm 0,51$ \\
\hline
\end{tabular}

Lxf $=$ Longueur du grand axe $; 1 \mathrm{xf}=$ Longueur du petit axe $; \mathrm{Pfr}=$ poids du fruit $; \mathrm{Ppu}=$ Poids de la pulpe $; \mathrm{Pgr}=$ Poids de la graine

Tableau 5 : Caractéristiques des feuilles et folioles de B. aegyptiaca selon les sites.

\begin{tabular}{lcccc}
\hline \multicolumn{5}{c}{ Sites } \\
\hline $\begin{array}{l}\text { Descripteur } \\
\text { morphologique }\end{array}$ & Arkou & Kokorguin & Mandafana & Moyenne \\
\hline Lfo $(\mathrm{mm})$ & $35,54 \pm 0,77$ & $35,93 \pm 0,57$ & $36,02 \pm 0,59$ & $35,83 \pm 0,64$ \\
lfo $(\mathrm{mm})$ & $28,52 \pm 0,92$ & $25,65 \pm 0,86$ & $27,18 \pm 0,76$ & $27,11 \pm 0,84$ \\
Sfo $\left(\mathrm{mm}^{2}\right)$ & $1037,7 \pm 246,91$ & $945,22 \pm 243,94$ & $1003,0 \pm 285,04$ & $995,30 \pm 258,63$ \\
Lep $(\mathrm{cm})$ & $4,16 \pm 0,56$ & $3,63 \pm 0,36$ & $4,44 \pm 0,45$ & $4,07 \pm 0,45$ \\
\hline Lfo $=$ Longueur des folioles $;$ lfo $=$ Largeur des folioles $;$ Sfo $=$ Surface des folioles $;$ Lep $=$ Longueur des épines
\end{tabular}

\section{DISCUSSION}

L'étude a permis de comprendre que les populations de la zone d'étude disposent d'amples connaissances sur le $B$. aegyptiaca. D'une manière générale, le nombre de critères utilisés pour classer l'espèce a varié selon le groupe ethnique. Cependant, les Maba utilisent plus de critères (13 critères) que les autres groupes. Les critères utilisés par les différents groupes ethniques pour distinguer les individus de l'espèce sont liés à la taille des arbres, la morphologie des feuilles, des fruits et au goût de la pulpe. Les critères communs aux quatre (4) groupes ethniques ont été la taille de l'arbre, les dimensions et la forme $\mathrm{du}$ fruit, le goût de la pulpe et la taille des feuilles. Cependant, le nombre de critères utilisés par chaque groupe pour différencier les individus de l'espèce ont été faibles comparés à ceux utilisés ailleurs (Assogbadjo, 2008; Fondohan, 2010 ; Sinsin, 2010) pour identifier d'autres espèces. Les descripteurs morphologiques quantitatifs de l'espèce ont permis de décrire et d'analyser les morphotypes identifiés ou non par les paysans. Dans cette zone agroécologique, les descripteurs morphologiques discriminants ont été la hauteur de l'arbre, la longueur du grand axe $\mathrm{du}$ fruit et la largeur des feuilles. La longueur du grand axe du fruit et la hauteur de l'arbre d'une part, et la largeur de la feuille et la longueur du fruit d'autre part, ont été significativement et positivement corrélées. Cela signifie que les individus de grande taille et qui portent de larges feuilles produisent de longs fruits. Ces descripteurs constituent donc des critères de différenciation entre les individus de l'espèce. Très influencés par les facteurs du milieu, ces critères peuvent être par conséquent intégrés dans le choix de la sélection des variétés pour les futurs programmes de domestication basée sur les connaissances endogènes. En effet, l'existence de la variabilité phénotypique au sein et entre les populations naturelles constitue la base de la sélection et de l'amélioration variétales (Simons et Leakey, 2004). 
La distribution de B. aegyptiaca dans la Région du Ouaddaï n'est pas homogène. La majorité des personnes enquêtées affirme que B. aegyptiaca se développent beaucoup plus sur les plaines et les sols sableux. Ces résultats confirment ceux de Ndong et al. (2015) qui ont localisé des groupements de $B$. aegyptiaca sur des substrats identiques. Bien qu'ils soient en nombre réduit, ces trois (3) morphotypes identifiés sont conformes à la classification du groupe ethnique Maba. Le morphotype intéressant pour des programmes de sélection et de domestication de cette espèce est le morphotype qui regroupe des arbres de grande taille qui portent des feuilles larges et qui produisent de longs fruits.

Les résultats de la présente étude ont montré que les populations locales distinguent des individus de $B$. aegyptiaca à tronc lisse et ceux à tronc rugueux. Ces observations sont conformes à celles de Sagna et al. (2014) qui ont fait la même observation sur la texture (lisse ou rugueuse) du tronc chez l'espèce. Ces auteurs rapportent que les troncs de $B$. aegyptiaca sont le plus souvent lisses chez les jeunes individus avant de devenir plus rugueux à l'âge adulte. La texture du tronc des individus serait probablement liée à leur âge. Toutefois, ces caractéristiques ne peuvent être utilisées dans le cadre de la classification taxonomique car les distinctions sont souvent imprécises, donc peu fiable. Le diamètre à hauteur de poitrine chez l'espèce a varié entre 16,63 et 19,09 $\mathrm{cm}$ et la hauteur moyenne de la première branche vivante a oscillée entre 1,86 et $2,95 \mathrm{~m}$. Ces variations ne sont qu'apparentes, étant donné qu'il n'existe pas de différence significative entre le diamètre des individus et la hauteur de leur première ramification basale. Ces résultats ont montré que les populations de B. aegyptiaca bien que situées à différentes altitudes, ont présenté un même diamètre à hauteur de poitrine et une même hauteur de la première grosse branche. Cela signifie aussi que ces deux caractères ne sont pas influencés par l'environnement mais sont régis par le mécanisme des gènes. Ces caractères ne sauraient être des critères de différenciation pour l'espèce. En revanche, une grande variabilité a été observée chez d'autres espèces de savanes sèches (Kouyaté, 2005).

La hauteur de la première branche vivante est un caractère qui renseigne sur l'accessibilité à la canopée de l'arbre (Mapongmetsem et al., 2011). Elle ne constitue pas en revanche un critère de différenciation pour les individus de $B$. aegyptiaca. D'autres études (Kouyaté et Van Damme, 2002; Abasse et al., 2011) ont aussi trouvé de grandes variabilités morphologiques chez d'autres espèces de savanes sèches.

Les fruits ont présenté un poids moyen de variabilité restreinte $(4,90$ à $5,04 \mathrm{~g})$. Ces résultats sont contraires à ceux d'Abasse et al. (2011) qui ont montré une grande variabilité dans le poids et la morphologie des fruits de B. aegyptiaca. Ces observations sont en revanche similaires à celles de Soloniev et al. (2004) qui ont obtenu des poids moyens des fruits de l'ordre de 4,04 $\pm 0,64 \mathrm{~g}$.

La moyenne des poids des pulpes a été de $1,87 \pm 0,65 \mathrm{~g}$. Ce poids est également comparable à celui observé par Soloviev et al. (2004) avec une faible variabilité entre les accessions. Ces auteurs ont également montré que le poids du fruit varie significativement selon la zone écologique. D'une manière générale, les fruits de B. aegyptiaca étudiés ont présenté un rapport pondéral (Pulpe/fruit) qui est de $37 \%$ du poids total du fruit. Ce taux peu élevé est nettement inférieur au taux de 59\% obtenus par Abasse et al. (2011).

Les résultats de la caractérisation des feuilles et folioles de B. aegyptiaca ont montré que leurs dimensions (longueur, largeur et surface) n'ont pas significativement varié. Ces résultats montrent que la longueur, la largeur ainsi que la surface des feuilles ne constituent pas des critères de différenciation entre les individus de cette espèce. Cela s'explique par le fait que le caractère dimensions de la feuille n'est pas sous l'influence du milieu mais est plutôt contrôlé par les gènes.

\section{Conclusion}

L'étude a montré que les populations de la zone d'étude ont une bonne connaissance sur le B. aegyptiaca. Pour différencier les individus, les critères communs utilisés par les 4 groupes ethniques ont été la taille de l'arbre, la grosseur et la 
forme du fruit, le goût de la pulpe et les dimensions des feuilles. Ces critères utilisés restent cependant quantitatifs mais pourront en revanche aider dans l'identification d'individus productrices de graines et à la connaissance des caractéristiques morphologiques nécessaires à la domestication de l'espèce. Les descripteurs quantitatifs retenus ont permis de distinguer parmi les morphotypes identifiés, des individus qui présentent une grande importance pour la mise sur pied des programmes de sélection et de domestication de l'espèce. Il s'agit des individus de $B$. aegyptiaca de grande taille à feuilles larges et aux longs fruits. Les connaissances endogènes constituent donc une source d'inspiration dans le choix des techniques à mettre en œuvre pour aboutir à la domestication de cette espèce. Les informations recueillies au terme de ce travail pourraient être utilisées dans le cadre de la sélection des ligneux qui répondent aux préoccupations des communautés locales. De plus, l'étude gagnerait à être complétée par d'une part, des observations sur l'utilisation et l'état de conservation de l'espèce et, d'autre part, des études sur les propriétés organoleptiques et biochimiques des parties consommées de l'arbre dans toutes les régions du Tchad.

\section{CONFLIT D'INTÉRÊTS}

Les auteurs déclarent qu'aucun conflit d'intérêts ne les oppose.

\section{CONTRIBUTIONS DES AUTEURS}

En soumettant le manuscrit pour une éventuelle publication dans votre journal, les auteurs reconnaissent et déclarent solennellement avoir pris activement part (chacun selon ses compétences et son grade) au travail de rédaction de l'article de façon substantielle.

BA a effectué ses travaux dans le cadre de son mémoire de Master II à l'université de Ngaoundéré. C'est le concepteur du projet de recherche et des méthodes de travail. Les collectes des données sur le terrain ayant abouti aux résultats ont été assuré par lui. Il a pris aussi activement part à l'analyse ainsi qu'à l'interprétation des résultats jusqu'à la rédaction de l'article de recherche. Il a enfin approuvé la version finale du manuscrit soumis à la revue pour publication. Son concours intellectuel a été d'un très important apport.

$\mathrm{ABB}$ a assuré le suivi des travaux de terrain et a contribué simultanément à la conception de la recherche et des méthodes de travail, à l'analyse et à l'interprétation des résultats. Il a aussi pris activement part à la rédaction de l'article, la révision critique du manuscrit avec un apport intellectuel important jusqu'à l'approbation de la version finale.

PMM a suivi de bout en bout ces activités de recherche dès leur conception jusqu'aux résultats finaux. A ce titre, il a aussi pris activement part à la rédaction de l'article, la lecture et la relecture critique du manuscrit avec un apport intellectuel très important jusqu'à l'approbation de la version finale.

\section{REFERENCES}

Abasse T, Weber JC, Katkore B, Boureima M, Larwanou M, Kalinganire A. 2011. Morphological variation in B. aegyptiaca (L.) Del. Fruits and seeds withing and among parkland agroforests in eastern Niger. Agrofor. Syst., 81: 57-66. DOI : 10.1007/s10457-010-9323-x

Arbonnier M. 2004. Arbres, Arbustes et Lianes des Zones Sèches d'Afrique de l'Ouest (2 ${ }^{\text {ème }}$ édn). CIRAD-MNHN ; 541 p.

Assogbajo AE, Kyndt T, Chadre FJ, Sinsin B, Gheysen G, Eyog-Matig O, Van Damme P. 2008. Genetic fingerprinting using AFLP cannot distinguish traditionally classified baobab morphotypes. Agroforestry Systems, 75: 157-165. DOI: 10.1007/s10457-010-9323-x

Chevalier MH, Vaillant A, Bensaid S, ShkiBoutamine R, Diallo OB, Sanou J, Bouguedoura N, Babin D. 2004. Impacts des pratiques humaines sur la conservation et la gestion in situ des ressources forestières: cas d'Acacia tortilis subs. raddiana et de Balanites aegyptiaca CSFD. Rapport du projet $\mathrm{n}^{\circ} 57$ $68 \mathrm{p}$.

Elfeel AA. 2010. Variability in Balanites aegyptiaca var. aegyptiaca seed kernel 
oil, protein and minerals contents between and within locations. Agric. Biol. J. North Am, (ABJNA), 1(2): 170-174. DOI: http://www.scihub.org/ ABJNA/index.html

Elseed AMAF, Amin AE, Khadiga AA, Ali J Sekene, Hishinum M, Hamana K. 2002. Nutritive evaluation of some fodder tree species during the dry season in Central Soudan. Asian-Aust. J. Anim. Sci., 15(6): 844-850. DOI: http://dx.doi.org/ 10.5713/ajas.2002.844

Eyog MO, Gande G, Dossou B. 2000. Programme des ressources génétiques forestières en Afrique au sud du Sahara. Compte rendu de la première réunion. Ouagadougou, Réseau «Espèces Ligneuses Alimentaires », 243 p.

Fandohan B, Assogbadjo AE, Kakaï RG, Kyndt T, Sinsin B. 2010. Quantitative descriptors confirm traditionally classified morphotypes of Tamarindus indica L. fruits. Faculty of Bioscience Engineering, University of AbomeyCalavi, Bénin. Fruits, 65 : 11-19. DOI: 10.1051/fruits:2005039

FAO (Food and Agriculture Organization), 2001. Situation des ressources génétiques forestières du Nord Cameroun. Archives et documents de la FAO. Département forêt. Rome. www.fao.org

INSEED (Institut National des Statistiques et des Etudes Economiques). 2009. Deuxième Recensement Général de la Population et de l'Habitat, Résultats Généraux.

Kouyaté AM, Van Damme P. 2002. Caractères morphologiques de Detarium microcarpum Guill. et Perr. au sud du Mali. Fruits, 57 : 231-238. DOI : 10.1051/fruits:2005039

Kouyaté AM, 2005. Aspects ethnobotaniques et étude de la variabilité morphomogique, biochimique et phénologique de Detarium microcarpum Guill. \& Perr. au Mali. Thèse, Université de Gand, Belgique, p.180.

Makalao MM, Savadogo A, Zongo C, Traoré AS. 2015. Composition nutritionnelle de 10 fruits sauvages consommés dans trois Départements du Tchad. Int. J. Biol.
Chem. Sci., 9(5): 2385-2400. DOI: 10.4314/ijbcs.v9i5.11

Mapongmetsem PM, Kapchie VN, Tefempa BH. 2012. Diversity of local fruit trees and their contribution in sustaining the rural livelihood in the Northern Cameroon. Ethiopian Journal of Environmental studies and Management, 5(1): 37-43. DOI : 10.4314/ejesm.v5i1.5

Mapongmetsem PM, Nkongmenek AB, Romgoumi G, Dongock ND, Dongmo B. 2011. Impact des systèmes d'utilisation des terres sur la conservation de Vitellaria paradoxa Gaertn. F. (Sapotaceae) dans la region des savanes soudano-guinéennes. International Journal of Environmental Studies, 68(6): 851-872. DOI: 10.1080/00207233.2011.641238

Ndong AT, Ndiaye O, Sagna MB, Diallo A, Galop D, Guisse A. 2015. Caractérisation de la végétation ligneuse sahélienne du Sénégal: cas du Ferlo. Int. J. Biol. Chem. Sci., 9(6): 2582-2594; DOI: 10.4314/ijbcs.v9i6.6

Sagna MB, Diallo A, Sarr PS, Ndiaye O, Goffner D, Guisse A. 2014. Biochemical composition and nutritional value of Balanites aegyptiaca (L.) Del fruit pulps from Northern Ferlo in Senegal. African Journal of Biotechnology, 13(2): 336342. DOI: 10.5897/AJB2013.12395

Simons AJ, Leakey RRB. 2004. Tree domestication in tropical agroforestry. Agrofor Syst., 61: 167-181. DOI: 10.1007/s10457-010-9323-x

Sinsin B. 2010. How far local use of taget plant species challenge their conservation in land use area in West Africa. Com. Internatioal Sympo. On indigenous fruit trees for dry land Africa. Domestication for use in changing environment Allada, Benin, $12 \mathrm{p}$.

Soloniev P, Niang TD, Gaye A, Totte A. 2004. Variabilité des caractères physicochimiques des fruits de trois espèces ligneuses de cueillette, récoltés au Sénégal: Adansonia digitata, Balanites aegyptiaca et Tamarindus indica. Fruit, 59: $109-119 . \quad$ DOI : 10.1051/fruits:2004011 